AperTO - Archivio Istituzionale Open Access dell'Università di Torino

First in vivo MRI study on theranostic dendrimersomes

This is a pre print version of the following article:

Original Citation:

Availability:

This version is available http://hdl.handle.net/2318/1633038

since 2017-05-11T09:01:19Z

Published version:

DOI:10.1016/j.jconrel.2017.01.010

Terms of use:

Open Access

Anyone can freely access the full text of works made available as "Open Access". Works made available under a Creative Commons license can be used according to the terms and conditions of said license. Use of all other works requires consent of the right holder (author or publisher) if not exempted from copyright protection by the applicable law. 


\section{Accepted Manuscript}

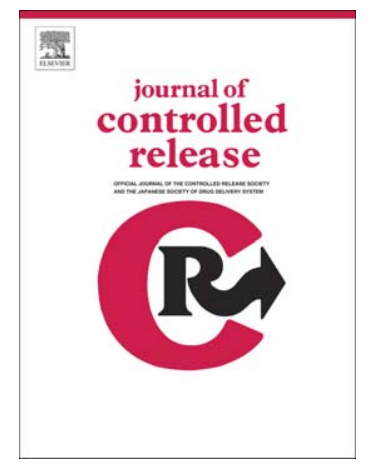

DOI:

S0168-3659(16)31017-3

Reference:

doi:10.1016/j.jconrel.2017.01.010

To appear in: Journal of Controlled Release

Received date: 24 October 2016

Revised date: 26 December 2016

Accepted date: $\quad 4$ January 2017

Please cite this article as: Miriam Filippi, Valeria Catanzaro, Deyssy Patrucco, Mauro Botta, Lorenzo Tei, Enzo Terreno, First in vivo MRI study on theranostic dendrimersomes, Journal of Controlled Release (2017), doi:10.1016/j.jconrel.2017.01.010

This is a PDF file of an unedited manuscript that has been accepted for publication. As a service to our customers we are providing this early version of the manuscript. The manuscript will undergo copyediting, typesetting, and review of the resulting proof before it is published in its final form. Please note that during the production process errors may be discovered which could affect the content, and all legal disclaimers that apply to the journal pertain. 


\title{
First in vivo MRI Study on Theranostic Dendrimersomes
}

Miriam Filippi ${ }^{\mathrm{a}}$, Valeria Catanzaro ${ }^{\mathrm{a}}$, Deyssy Patrucco ${ }^{\mathrm{a}}$, Mauro Botta ${ }^{\mathrm{b}}$, Lorenzo Tei ${ }^{\mathrm{b}}$, and Enzo Terreno $^{\mathrm{a} *}$

${ }^{\text {a }}$ Centro di Imaging Molecolare e Preclinico, Dipartimento di Biotecnologie Molecolari e Scienze della Salute, Università di Torino, Via Nizza 52, 10126, Torino, Italia.

E-mail addresses: miriam.filippi@unito.it, valeria.catanzaro@unito.it, deyssyj@gmail.com. *Corresponding author: Enzo Terreno. E-mail address: enzo.terreno@unito.it; Telephone: +39 011 6706452; Fax: +39 0116706487.

${ }^{\mathrm{b}}$ Dipartimento di Scienze ed Innovazione Tecnologica, Università del Piemonte Orientale "Amedeo Avogadro", Viale T. Michel 11, 15121, Alessandria, Italia.

E-mail addresses: mauro.botta@uniupo.it, lorenzo.tei@uniupo.it. Telephone: +390131 360 208; Fax: +390131360250.

\begin{abstract}
Amphiphilic Janus-dendrimers are able to self-assemble into nanosized vesicles named dendrimersomes. We recently synthesized the $3,5-\mathrm{C}_{12}-\mathrm{EG}-(\mathrm{OH})_{4}$ dendrimer that generates dendrimersomes with very promising safety and stability profiles, that can be loaded with different contrast agents for in vivo imaging. In this contribution, nanovesicles were loaded with both the Magnetic Resonance Imaging (MRI) reporter $\operatorname{GdDOTAGA}\left(\mathrm{C}_{18}\right)_{2}$ and the glucocorticoid drug Prednisolone Phosphate (PLP), in order to test their effective potential as theranostic nanocarriers on murine melanoma tumour models. The incorporation of $\operatorname{GdDOTAGA}\left(\mathrm{C}_{18}\right)_{2}$ into the membrane resulted in dendrimersomes with a high longitudinal relaxivity $\left(r_{1}=39.1 \mathrm{mM}^{-1} \mathrm{~s}^{-1}\right.$, at $310 \mathrm{~K}$ and 40 $\mathrm{MHz}$ ) so that, after intravenous administration, $\mathrm{T}_{1}$-weighted MRI showed a consistent contrast enhancement in the tumour area. Furthermore, the nanovesicles encapsulated PLP with good efficiency and displayed anti-tumour activity both in vitro and in vivo, thus enabling their practical use for biomedical theranostic applications.
\end{abstract}

\section{Keywords}

Dendrimersomes, prednisolone phosphate, melanoma, theranostic, magnetic resonance imaging. 


\section{Introduction}

Despite the rapid advances in diagnostic and therapeutic protocols, cancer still remains one of the most challenging domains of investigation in modern medicine. The limitations or the failure of current treatments encouraged the research for innovative approaches to cancer disease, in the effort to improve the early-stage detection and specifically target the therapy. ${ }^{1}$

By combining both therapeutic and diagnostic properties in one single platform, theranostic systems are emerging medical tools that hold great promises in enhancing the therapeutic outcome of cancer therapy. ${ }^{2-5}$ Unlike conventional approaches, the theranostics integrates specific molecular targeting, therapeutic activity, and imaging in one multifunctional medical system, very often based on the use of nanosized carriers. Some of the most attractive features of the nanoparticle-based theranostics include the opportunity to specifically deliver different pharmaceutically active molecules, to monitor their biodistribution in vivo, and, in some cases, also to control/trigger the release of the drug at the target site. ${ }^{5,6}$

So far, a very wide and continuously extending array of nanoplatforms has been considered for biomedical applications, but few of them have received so much interest in the theranostic field as liposomes. These vesicles, composed of a phospholipidic bilayer, already proved their ability to load both imaging-responsive molecules and drugs, and to act as theranostic agents in several pathologic scenarios, especially in oncology.

Following the work on liposomes, other nanosized bilayered vesicular structures have been proposed. Among them, dendrimersomes (DSs) are vesicles self-assembled from amphiphilic Janus-dendrimers, described for the first time by Percec et al. in $2010 .^{8}$ Based on these initial studies, we argued whether DSs could be employed as reliable alternative to liposomes in the advanced biomedical protocols, especially considering the advantages in terms of cost and easiness of synthesis of the dendrimeric constituents of the membrane. Therefore, we initially evaluated the potential of these nanocarriers to act as MRI agents by loading paramagnetic Gd-complexes either in the aqueous core or in the membrane of the particles. ${ }^{9-11}$ Furthermore, we recently synthesized a 
new low generation Janus-dendrimer $\left(3,5-\mathrm{C}_{12}-\mathrm{EG}-(\mathrm{OH})_{4}\right.$, Chart 1, left $)$ that has been demonstrated to form Gd-loaded dendrimersomes of high stability and biocompatibility. ${ }^{10}$

In this contribution, we report on the first in vivo testing of the theranostic potential of DSs composed of the 3,5- $\mathrm{C}_{12}-\mathrm{EG}-(\mathrm{OH})_{4}$ dendrimer, co-loaded with a drug (Prednisolone Phosphate, PLP, Chart 1) and the lipophilic MRI agent GdDOTAGA $\left(\mathrm{C}_{18}\right)_{2}$ (Chart 1), whose excellent MRI performance has been very recently demonstrated. ${ }^{11}$ Since liposomal formulations of PLP already proved to be effective in the treatment of melanoma, ${ }^{12-18}$ the herein investigated theranostic platform was tested in vivo on a syngeneic murine melanoma model and its therapeutic efficacy was compared to that provided by a corresponding liposome-based formulation.
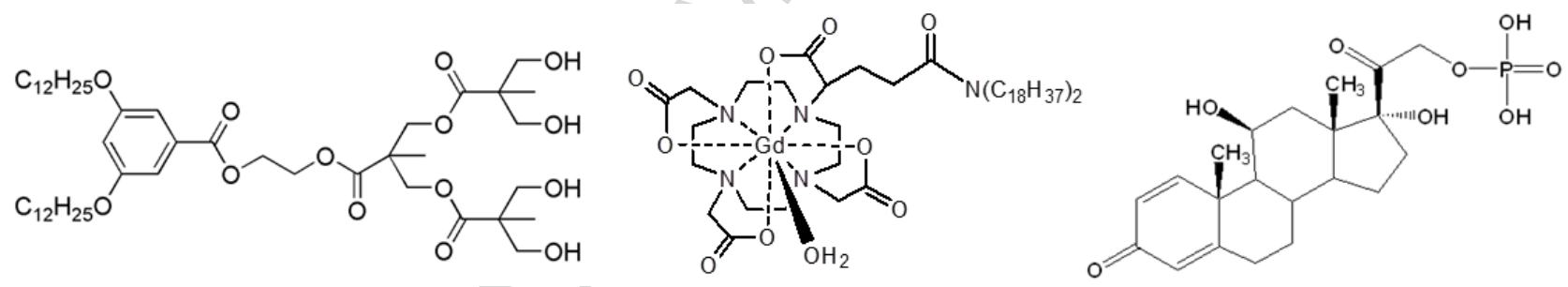

Chart 1. Structures of the dendrimersomes components. Left: 3,5-C12-EG- $(\mathrm{OH})_{4}$ Janus dendrimer (main constituent of the dendrimersomes); middle: GdDOTAGA(C18) $)_{2}$ complex (MRI agent incorporated in the dendrimersome bilayer); right: prednisolone phosphate (PLP, antitumour drug encapsulated in the inner core of the dendrimersomes).

\section{Materials and methods}

\section{Chemicals}

The phospholipids 1,2-distearoyl-sn-glycero-3 phosphoethanolamine-N[carboxy(polyethyleneglycol)-2000] ammonium salt (DSPE-PEG2000-COOH) and 1,2dipalmitoyl-sn-glycero-3-phosphocholine (DPPC) were purchased from Avanti Polar Lipids Inc. (Alabaster, AL, USA). The 3,5- $\mathrm{C}_{12}-\mathrm{EG}-(\mathrm{OH})_{4}$ Janus dendrimer was synthesized as described elsewhere. ${ }^{10}$ Briefly, the amphiphile was obtained by linking the hydrophobic block 3,5 bis-dodecyl substituted benzoyl ether to a generation 1 of 2,2-bis(hydroxymethyl)propanoic acid, using ethylene glycol as spacer between the two moieties. The first synthesis intermediate, a 2- 
hydroxyethyl 3,5-(didodecyloxy)benzoate was obtained by reacting 3,5-(didodecyloxy)benzoic acid with an excess of ethylene glycol in the presence of 4-(dimethylamino)pyridinium ptoluenesulfonate (DPTS) and dicyclohexylcarbodiimide (DCC). Following the same coupling procedure, 2,2,5-trimethyl-1,3-dioxane-5-carboxylic acid was reacted with the free hydroxyl group of the first synthesis intermediate. Deprotection of the acetonide was carried out in a 1:1 mixture of $6 \mathrm{M} \mathrm{HCl}$ and THF to get a generation zero intermediate. The final compound was obtained by repeating once again the coupling and the deprotection steps.

$\operatorname{GdDOTAGA}\left(\mathrm{C}_{18}\right)_{2}$ was synthesized in a four steps-process starting from the DOTAGA $(t \mathrm{Bu})_{4}$, as it was already reported in literature. ${ }^{11}$ Briefly, the free carboxylic acid of DOTAGA $(\mathrm{tBu})_{4}$ was activated by formation of the $N$-hydroxysuccinimidyl ester, which was then reacted with dioctadecylamine in pyridine at $70^{\circ} \mathrm{C}$. The free ligand was obtained after $t \mathrm{Bu}$ esters deprotection using a 1:1 mixture of TFA and dichloromethane and the Gd(III) complex was prepared by reacting the ligand with $\mathrm{GdCl}_{3}$ in methanol at $50^{\circ} \mathrm{C}$ overnight. All other chemicals and reagents were purchased from Sigma-Aldrich (St. Louis, USA), and used as received unless otherwise noted.

\section{Nanovesicles preparation}

DSs and liposomes were prepared by the thin film hydration method. ${ }^{19}$ Briefly, to prepare the stock samples with a concentration of $80 \mathrm{mg} / \mathrm{ml}$ in the final vesicle suspension useful to perform further experiments, the appropriate amounts of amphiphilic materials $\operatorname{GdDOTAGA}\left(\mathrm{C}_{18}\right)_{2}$, DSPEPEG2000-COOH, and 3,5- $\mathrm{C}_{12}-\mathrm{EG}-(\mathrm{OH})_{4}$ dendrimer (or the phospholipid DPPC) were weighted according to the molar ratio 20:5:75, and then dissolved into chloroform (8 ml). After dissolution, the organic solvent was slowly evaporated, so that the amphiphilic components distributed in a thin homogeneous film at the bottom of a round flask. After two hours under vacuum, the film was hydrated at $50^{\circ} \mathrm{C}$ with an isotonic buffered solution containing PLP at $50 \mathrm{mg} / \mathrm{ml}$, in addition to $\mathrm{NaCl}$ and 4-(2-hydroxyethyl)-1- piperazineethanesulfonic acid (HEPES) with a final whole osmolarity of $c a .300 \mathrm{mOsm}$ and $\mathrm{pH}$ of 7.4. The vesicular suspension was then extruded several times through polycarbonate filters (Lipex extruder, Northern Lipids Inc.) with pore diameters 
decreasing from $1 \mu \mathrm{m}$ to $200 \mathrm{~nm}$. Finally, to completely remove the non-encapsulated drug, an exhaustive dialysis was carried out at $4^{\circ} \mathrm{C}$ against an isotonic buffer consisting of a $\mathrm{pH} 7.4$ solution $0.15 \mathrm{M}$ of $\mathrm{NaCl}$ and 0.004 of $\mathrm{M}$ 4-(2-hydroxyethyl)-1- piperazineethanesulfonic acid (HEPES), in a total volume of $2 \mathrm{~L}$ and for a minimal duration of 16 hours, with one buffer renewal after the first 4 hours.

\section{Nanovesicles characterization}

The mean hydrodynamic diameter and the polydispersity of the vesicles were determined by Dynamic Light Scattering (DLS, Zetasizer Nano 90 ZS, Malvern, UK).

The relaxometric characterization of $\operatorname{GdDOTAGA}\left(\mathrm{C}_{18}\right)_{2}$-bearing nanoformulations was carried out to define the longitudinal relaxivity $r_{1}$ (at $25^{\circ} \mathrm{C}$ and $37^{\circ} \mathrm{C}$ ) as a function of the applied magnetic field. Specific details about such measurements are available on the Electronic Supplementary Information (ESI). The total concentration of Gd in the samples was determined by a relaxometric measurement (at $20 \mathrm{MHz}$ ) after mineralization. Briefly, the samples were diluted 1:2 with concentrated $\mathrm{HCl}(37 \%)$ and stored overnight at high temperature $\left(120^{\circ} \mathrm{C}\right)$ in a sealed glass ampoule to ensure the complete release of the metal ions from the complexes and to obtain free Gd(III) aqua-ions. The $R_{1}{ }^{\text {obs }}$ measurement (at $25^{\circ} \mathrm{C}$ ) allowed for the accurate estimation of the $\mathrm{Gd}$ concentration through a calibration line obtained by using standard solutions of $\mathrm{GdCl}_{3}$.

The total amount of PLP loaded into the vesicles was measured by analytical high-performance liquid chromatography (HPLC), carried out on a Waters Alliance System (separations module: 2695 model) equipped with a photodiode array detector (model 2998) and a Waters Atlantis $\mathrm{C}_{18}$ reverse phase column $(4.6 \mathrm{~mm} \times 150 \mathrm{~mm}, 3.5 \mu \mathrm{m})$. The chromatographic separation took 37 minutes in gradient mode, with a re-equilibration time of $18 \mathrm{~min}$ to restore the initial conditions. Gradient composition started from $95 \%$ of $\mathrm{A}\left(\mathrm{H}_{2} \mathrm{O}\right.$ containing $0.1 \%$ Trifluoro Acetic Acid, TFA)-5\% of B $\left(\mathrm{CH}_{3} \mathrm{CN}\right.$ added with $0.1 \%$ TFA) and it was maintained for $2.5 \mathrm{~min}$. Then, B was raised to $30 \%$ at 15 min, $60 \%$ at $30 \mathrm{~min}$, and finally $100 \%$ at $35 \mathrm{~min}$ remaining until the end of the analysis. The total amount of PLP in the vesicular suspension $(5 \mathrm{mg}$ of amphiphilic material per $\mathrm{mL}$ of hydration 
solution) was quantified by monitoring the eluate through UV detection (detection wavelengths set between 200 and $400 \mathrm{~nm}$ ). The drug quantification was performed at $\lambda=278 \mathrm{~nm} .^{20}$ In order to determine the drug-loading efficiency of the nanocarriers, the PLP content of samples was then compared to the initial amount of PLP present in the hydration solution (0.103 M).

\section{Cells}

All cell lines were obtained from American Type Culture Collection (ATCC, Manassas, VA, USA) and resulted negative for mycoplasma test MycoAlert $^{\mathrm{TM}}$ Mycoplasma Detection Kit, Lonza Sales AG, Verviers, Belgium). Murine fibroblasts (NIH/3T3), melanoma (B16.F10) and human umbilical vein endothelial (HUVEC) cells were cultured as monolayers at $37^{\circ} \mathrm{C}$ in a $5 \% \mathrm{CO}_{2}$-containing humidified atmosphere in Dulbecco's modified Eagle's medium (DMEM). The medium was supplemented with $10 \%(\mathrm{v} / \mathrm{v})$ of heat-inactivated foetal bovine serum, $2 \mathrm{mM}$ of Glutamine, 100 $\mathrm{U} / \mathrm{ml}$ of penicillin and $100 \mu \mathrm{g} / \mathrm{ml}$ of streptomycin. At $80 \%$ confluency, cells were trypsinized with $0.01 \%$ trypsin and $0.02 \%$ EDTA in Phosphate-buffered Saline (PBS). All cells employed for cellular tests were at passages included between 5 and 10. Culture medium, fetal bovine serum, penicillin-streptomycin mixture and trypsin were all purchased from Lonza (Lonza Sales AG, Verviers, Belgium).

\section{Cell viability assessment}

Cell viability was assessed on murine fibroblasts (NIH/3T3), melanoma (B16.F10) and human umbilical vein endothelial (HUVEC) cells after incubation with the paramagnetic dendrimersomes (Gd-DS), PLP-loaded paramagnetic dendrimersomes (PLP-Gd-DS) or liposomes (PLP-Gd-LIPO), and free PLP, after 1, 6 or $24 \mathrm{~h}$ incubation. The selected doses of PLP and amphiphilic material were 1 and $40 \mathrm{mg} / \mathrm{ml}$ respectively, in all experiments. Before being added to cell culture, vesicles or free PLP were diluted into culture medium to achieve the desired concentration and filtered by sterile $0.4 \mu \mathrm{m}$ filters. The viability was assessed by a fluorimetric assay based on the cell metabolic activity (CellTiter-Blue ${ }^{\circledR}$ Cell Viability assay, Promega, Madison, USA). The CellTiter-Blue ${ }^{\circledR}$ reagent contains the indicator dye Resazurin that is reduced to highly fluorescent Resofurin by 
viable cells retaining metabolic capacities, so that the fluorescent signal generated from the assay is proportional to the number of living cells in the sample. Briefly, NIH/3T3, HUVEC and B16.F10 cells were plated in 96-well multiwell plate at a concentration of $1.0 \times 10^{4}, 1.0 \times 10^{4}$ and $5.0 \times 10^{3}$ cells per well, respectively. After $24 \mathrm{~h}$, cells were exposed to the incubation medium for 1, 6 and $24 \mathrm{~h}$. Next, the incubation medium was removed and wells were washed three times with sterile PBS $(200 \mu \mathrm{l})$. The CellTiter-Blue ${ }^{\circledR}$ reagent was added following manufacturer's instructions $(20 \mu 1$ of reagent diluted 1:5 into culture medium for each well). After $4 \mathrm{~h}$, supernatants were collected and placed in a black 96-well multiwell plate for fluorescence detection (excitation wavelength: 530$560 \mathrm{~nm}$, emission wavelength: $590 \mathrm{~nm}$ ). Five independent experiments were performed to test each single condition, with cells plated in triplicate and fluorescence measurements repeated three times. The reported results represent the mean \pm SD of the percentage of viable cells, normalized with respect to the control samples obtained by incubating cells with PBS-containing medium.

\section{Animals}

Eight weeks old female C57/BL6 mice with similar weight (around 20-21 g) were used for the in vivo tests, with at least eight animals per condition in all performed experiments. All mice involved in the study were obtained from Charles River Laboratories (Calco, LC, Italy) and maintained in standard housing conditions, with water and standard rodent chow ad libitum and $12 \mathrm{~h}$ light/dark cycle. Animal manipulation and all experimental procedures were performed in accordance with the European Community guidelines and approved by the Italian Ministry of Health.

\section{In vivo MRI experiments}

The performance of the theranostic probes was investigated in mice bearing a syngeneic xenograft B16 melanoma tumour. Tumours were generated by subcutaneous injection of $2.5 \times 10^{5}$ melanoma cells (in $150 \mu \mathrm{l}$ of PBS) on the flank of the animal. After 10 days, mice developed solid tumours of 2-4 $\mathrm{mm}$ in diameter, and were ready to begin the treatment and the imaging procedures. For the MRI experiments, mice were anesthetized by intramuscular injection of a combination of tiletamine/zolazepam (zoletil 100; Virbac, Milan, Italy) $20 \mathrm{mg} / \mathrm{kg}$ and xylazine (Rompum; Bayer, 
Milan, Italy) $5 \mathrm{mg} / \mathrm{kg}$. Animals were administered via tail vein injection with PLP-Gd-DS or PLPGd-LIPO, receiving $0.04 \mathrm{mmol} / \mathrm{kg}$ of $\mathrm{Gd}(\mathrm{III})$ and $10 \mathrm{mg} / \mathrm{kg}$ of drug. Control experiments were carried out by injecting the paramagnetic dendrimersomes (Gd-DS). As shown in Scheme 1, the administration was repeated twice over a time range of 2 weeks: at day 0 (corresponding to the tenth day after tumour inoculation) and at day 7. MRI was carried out immediately before, immediately after, and 1, 2, 4 and 6 days after each injection. An additional image acquisition was performed $6 \mathrm{~h}$ after the second administration.

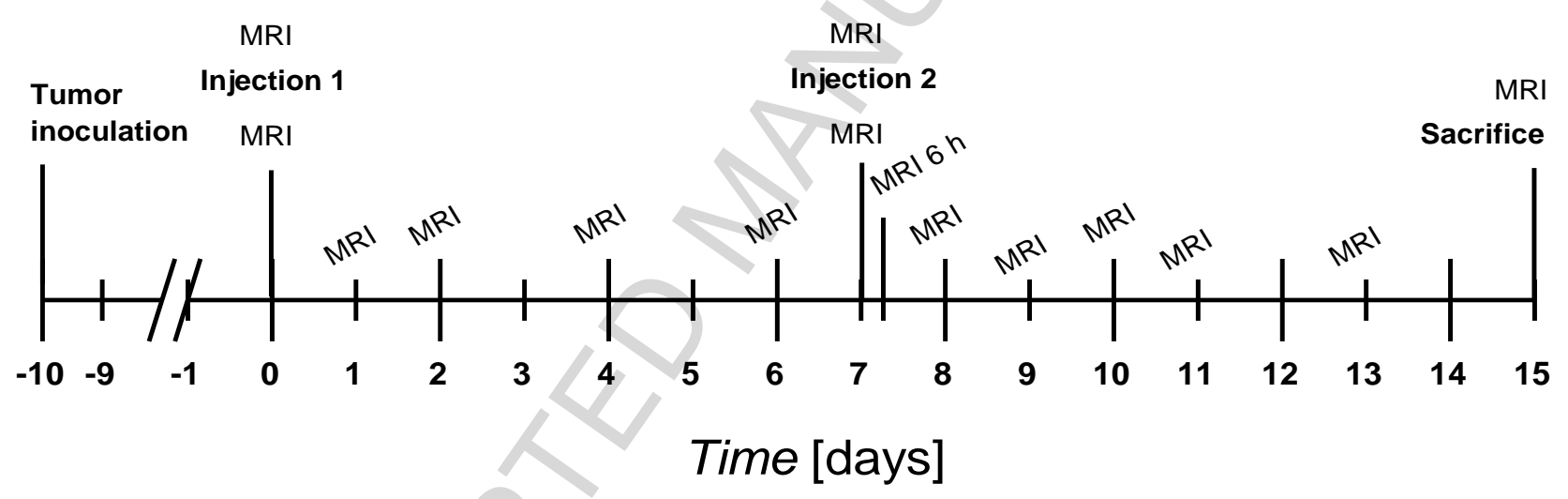

Scheme 1. Flowchart of the theranostic study. After tumour cells were subcutaneously inoculated, a time range of ten days was required for the tumour to develop. The nanoformulations loaded with Gd-DOTAGA $\left(\mathrm{C}_{18}\right)_{2}$ and PLP were injected at day 0 and day 7. MRI was carried out immediately before, immediately after and 24, 48, 96 and $144 \mathrm{~h}$ after each injection. An additional image acquisition was performed $6 \mathrm{~h}$ after the second administration. Finally, at day 15 animals were sacrificed.

Images were acquired on a 1 T MRI Scanner (Aspect M2 High Performance System, Aspect Magnet Technologies Ldt, Letanya, Israel), consisting of NdFeB Magnet equipped with a solenoid $\mathrm{Tx} / \mathrm{Tr}$ coil of inner diameter of $35 \mathrm{~mm}$. The system is equipped with a fast gradient (gradient strength $450 \mathrm{mT} \mathrm{m}^{-1}$ at $60 \mathrm{~A}$, ramp time $250 \mu \mathrm{s}$ at $160 \mu \mathrm{V}$ ), with a field homogeneity of 0.2-0.5 gauss. To evaluate the imaging performance of $\mathrm{DSs}, \mathrm{T}_{1}$-weighted images $\left(\mathrm{T}_{1 \mathrm{w}}\right.$-images $)$ were acquired by using a standard spin echo sequence, setting the following parameters: TR $=250 \mathrm{~ms}$, $\mathrm{TE}=8.9 \mathrm{~ms}$, matrix size $=128 \times 128$, slice thickness $=1.5 \mathrm{~mm}, \mathrm{NA}=8, \mathrm{FOV}=35 \mathrm{~mm}$. The Mean Signal Intensity $\left(S I_{0}\right)$ was calculated on $\mathrm{T}_{1 \mathrm{w}}$-images by manually drawing regions of interest (ROIs), 
and then it was normalized with respect to the signal calculated on a glass tube containing $0.5 \mathrm{mM}$ of Gadoteridol in aqueous solution, that was used as an external reference during image acquisition. The resulting value was the Normalized Signal Intensity, $\mathrm{SI}_{\mathrm{n}}$. The $\mathrm{T}_{1}$ contrast enhancement $\left(T_{1}{ }^{E n h}\right)$ was calculated from the normalized signal intensity values measured after the injection of the theranostic agent $\left(S I_{n}{ }^{P O S T}\right)$, after subtraction of the corresponding value measured before the injection $\left(S I_{n}{ }^{P R E}\right)$ :

$T_{1}^{E n h}=\frac{S I_{n}^{P O S T} S I_{n}^{P R E}}{S I_{n}^{P R E}}$

Multislice $\mathrm{T}_{2}$-weighted images were acquired by using a Fast Spin Echo sequence set with the following parameters: $\mathrm{TR}=2500 \mathrm{~ms}, \mathrm{TE}=49.4 \mathrm{~ms}$, matrix size $=160 \times 152$, slice thickness $=1.5$ $\mathrm{mm}, \mathrm{NA}=6, \mathrm{FOV}=40 \mathrm{~mm}$. The tumour volume was determined by accurately drawing a ROI along the perimeter of the lesion in each $\mathrm{T}_{2 \mathrm{w}}$-image, and by simply adding all slice volumes. The tumour growth was monitored for 15 days, and then the blood and the urine were sampled immediately after the final MRI acquisition. Finally, the animals were sacrificed and their organs (kidney, liver, spleen, muscle, heart, lung, and tumour) were explanted. In a special cohort of animals ( $n=8$ per condition), the sampling of blood and urine, and the organ explant were performed on day 7, without providing the second administration of PLP-nanovesicles. The content of Gd(III) in the various tissues was measured by the inductively coupled plasma mass spectrometry (ICPMS) after digestion. Briefly, the volume of blood and urine samples and the weights of all organs were registered. Blood samples were added with heparin $(20 \mu \mathrm{l})$ in order to avoid clotting and allow a precise quantification of the collected volume. Then, all biological specimens were treated with concentrated $\mathrm{HNO}_{3}(70 \%)$ and digested by applying microwave heating (Milestone MicroSYNTH, Microwave labstation equipped with an optical fiber temperature control and HPR-1000/6M six position high-pressure reactor, Bergamo, Italy). After digestion, the remaining material was diluted in ultrapure water (final volume of $3 \mathrm{~mL}$ ) and further filtered with $0.2 \mu \mathrm{m}$ filters. Finally, samples were analysed by ICP-MS (Thermo Scientific ELEMENT 2 ICP-MS -Finnigan, Rodano, Milano, 
Italy). For urines, the values of $\mathrm{Gd}(\mathrm{III})$ content were reported as metal concentration, whereas for organs they were expressed with respect to the organ mass. In case of blood, these values were finally reported as circulating metal concentration by calculating the Total Blood Volume (TBV) for each animal from the average value reported for mice $(79 \mathrm{ml} / \mathrm{kg}){ }^{20}$

\section{Statistical analysis}

All data are presented as Mean Values \pm Standard Deviation, unless noted otherwise. Significance of the differences among the experimental conditions was identified by applying the Student $t$-test or ANOVA test, as indicated.

\section{Results}

In vitro characterization

By hydrating the amphiphilic films (GdDOTAGA $\left(\mathrm{C}_{18}\right)_{2}$, DSPE-PEG2000-COOH, and 3,5-C 12 -EG$(\mathrm{OH})_{4}$ in molar ratio 20:5:75) with an isotonic PLP-containing solution, vesicles with favourable physico-chemical properties for in vivo experiments were obtained. The suspensions were found to show a good dimensional uniformity (PDI values $<0.2$ ) and a mean hydrodynamic diameter of around $150 \mathrm{~nm}$ (Table S1, ESI). As previously reported, ${ }^{10}$ the incorporation of the paramagnetic GdDOTAGA $\left(\mathrm{C}_{18}\right)_{2}$ complex provided vesicles with high proton relaxivity, achieving values of $r_{1}$ around $39.1 \mathrm{mM}^{-1} \mathrm{~s}^{-1}$ at $40 \mathrm{MHz}$ and $310 \mathrm{~K}$ (Table S2 and Figure S1, ESI). Liposomes with a membrane composition analogue to DS in which DPPS replaced the $3,5-\mathrm{C}_{12}$-EG- $(\mathrm{OH})_{4}$ dendrimer showed similar characteristics, and were then chosen as control formulation in this study. The two nanoformulations displayed a similar PLP loading efficiency and were able to retain an almost equivalent fraction of PLP during the preparation $(0.34 \%$ and $0.29 \%$ of total PLP present in the suspensions of DS and LIPO, respectively, when prepared at $5 \mathrm{mg} / \mathrm{ml}$ of amphiphilic materials). 


\section{Cytotoxicity}

The potential capability of the theranostic dendrimersomes to hinder the cancer progression was tested in vitro on several cell cultures (Figure 1).
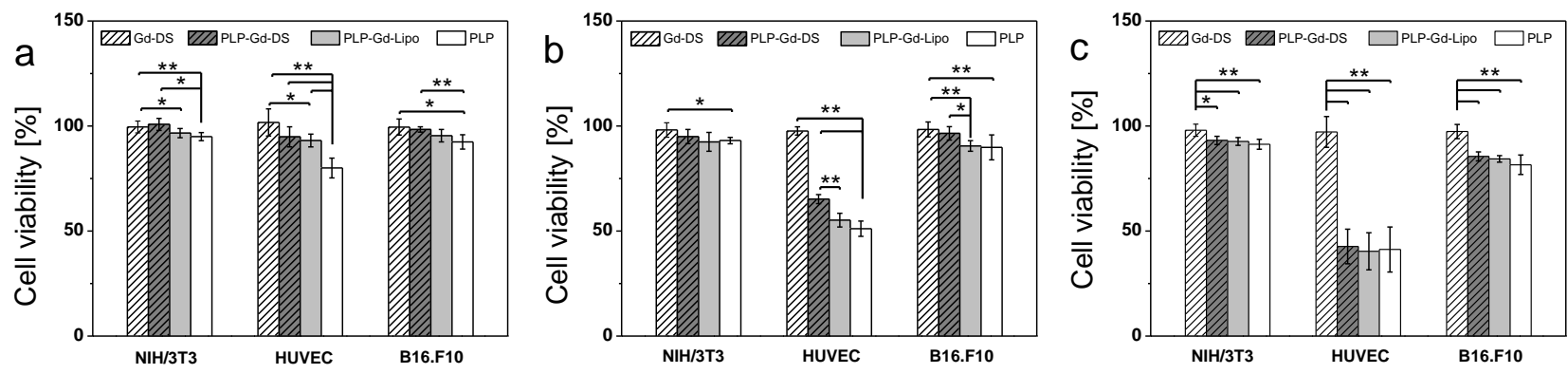

Figure 1. In vitro anti-tumour effects. Cell viability assessed by Resazurin/Resofurin assay on murine fibroblasts (NIH/3T3), melanoma (B16.F10) and human umbilical vein endothelial (HUVEC) cells after treatment with empty paramagnetic dendrimersomes (Gd-DS), paramagnetic PLP-loaded dendrimersomes (PLP-Gd-DS) or liposomes (PLPGd-LIPO), and free PLP. Incubation times were $1 \mathrm{~h}$ (a), $6 \mathrm{~h}$ (b) or $24 \mathrm{~h}$ (c). In all the experiments, the amounts of PLP and dendrimeric material were $1 \mathrm{mg} / \mathrm{mL}$ and $40 \mathrm{mg} / \mathrm{ml}$, respectively. Statistical significance addressed by ANOVA test (single and double asterisks indicating the $p$-value $<0.05$ and 0.01 , respectively).

In order to assess either the direct anti-tumour or the anti-angiogenic effects, melanoma cells (B16.F10 line, also used to generate the in vivo model), and endothelial cells (HUVEC line) were incubated with the control drug-free paramagnetic dendrimersomes (Gd-DS), the paramagnetic PLP-loaded dendrimersomes (PLP-Gd-DS), the correspondent liposomes (PLP-Gd-LIPO), and free PLP for 1,6 or $24 \mathrm{~h}$ at $37^{\circ} \mathrm{C}$. A fibroblast cell line (NIH/3T3) was employed as a control to identify possible unspecific cytotoxicity effects. In all conditions, the incubation was carried out so as to always deliver the same dose of PLP to the cells, and thereafter the cell viability was assessed. For all cell lines, the addition of the simple paramagnetic dendrimersomes at any incubation time range did not affect viability, thus indicating no toxicity associated with the presence of the vehicle in the medium. The free PLP dissolved into the medium induced only mild cytotoxicity on control fibroblasts and melanoma cells, with slightly more evident effects on melanoma cells at longer incubation times. However, the free PLP dramatically affected the endothelial cell viability, which 
resulted severely impaired even at short incubation times (with a decrease of $20.9 \%$ and $42.5 \%$ after 1 and $6 \mathrm{~h}$, respectively). After $1 \mathrm{~h}$ of incubation with the PLP-loaded nanosystems, only limited effects were observed: the maximum viability reduction $(\approx 8.6 \%)$ was detected on endothelial cells after incubation with PLP-Gd-LIPO, but all other data collected under these experimental conditions did not substantially differ. After $6 \mathrm{~h}$, the exposure to the nanosystems began to induce a stronger cytotoxicity on HUVECs and, though to a minor extent, also on B16.F10 cells. Interestingly, in both cases a statistically relevant difference ( $p$-value $<0.01$ and 0.05 for endothelial and melanoma cells, respectively) exists between the results obtained with PLP-Gd-DS and PLP-Gd-LIPO, with the latter showing a slightly higher effectiveness. Finally, for the longer incubation time $(24 \mathrm{~h})$, the efficacy of the two nanomedicines was almost equivalent, even if it varied depending on the tested cell line, being the endothelial and melanoma cell viability reduced to $40 \%$ and $85 \%$, respectively. Importantly, in these conditions the treatment with the PLPnanosystems always matched the results observed with the use of free PLP. Finally, it is arguable that some unspecific phenomena of cytotoxicity also occurred, as shown by the decrease of the viable fibroblast number ( $10 \%$ of viability decrease for both encapsulated and free drug).

In vivo MR imaging

In vivo $1 \mathrm{~T}$ MRI performed on melanoma-bearing mice after intravenous administration of PLP-GdDS revealed a consistent ( $c a .50 \%)$ contrast enhancement $\left(T_{1}^{E n h}\right)$ in the tumour area (Figure 2 and Figure 3) immediately after the first injection (day 0). 

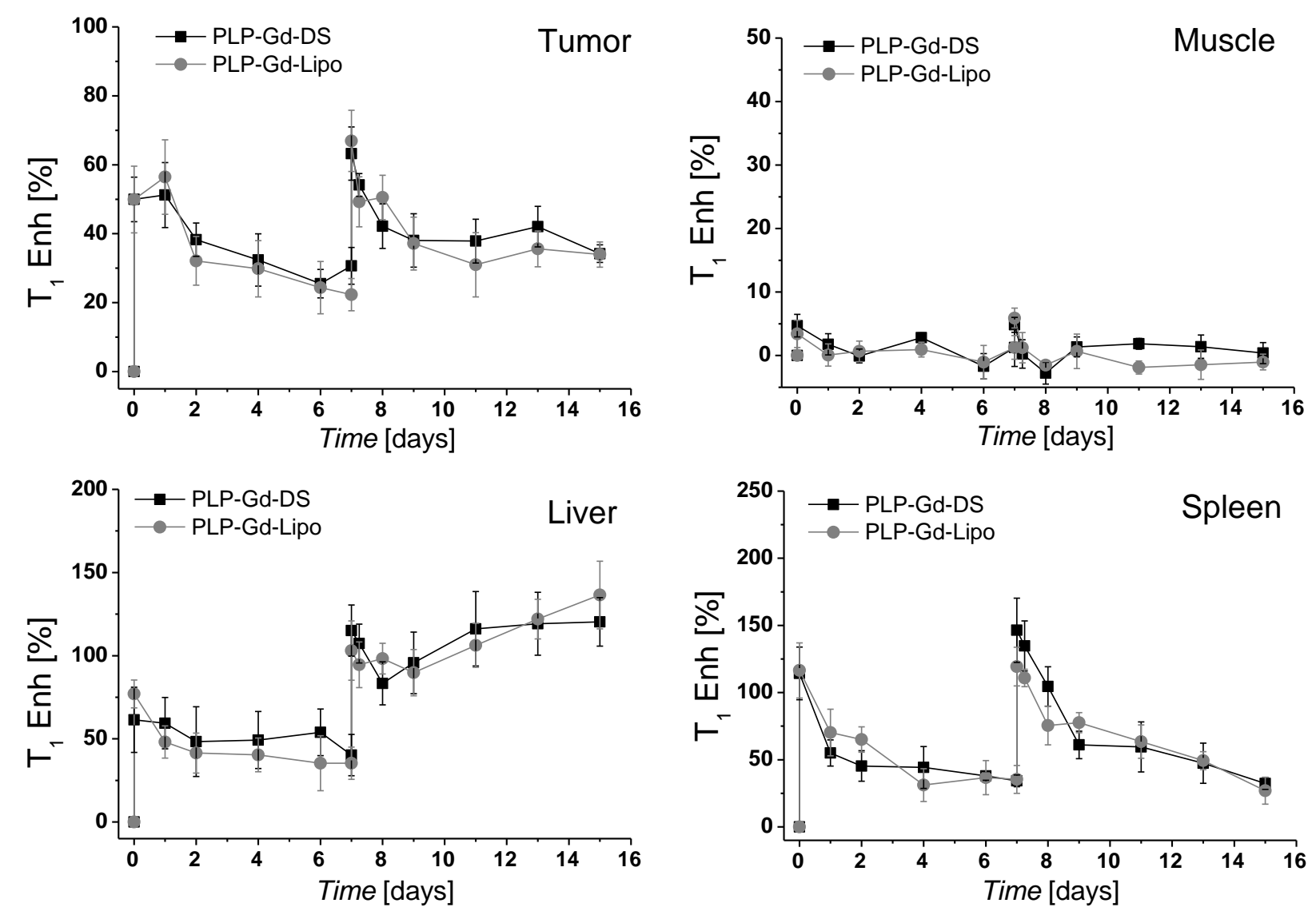

Figure 2. $\mathbf{T}_{1}$ contrast enhancement $\left(\boldsymbol{T}_{I}^{E n h}\right)$. Time evolution of the percentage $\mathrm{T}_{1}$ contrast enhancement $\left(T_{l}^{E N H}\right)$ calculated on $\mathrm{T}_{1 \mathrm{w}}$-images of tumour, muscle, liver and spleen after the systemic administration of PLP-loaded dendrimersomes (black squares) and liposomes (gray circles) bearing the Gd-complex $\operatorname{GdDOTAGA}\left(\mathrm{C}_{18}\right)_{2}$. The time evolution of $T_{l}^{E N H}$ observed on muscular tissue is reported as a negative control. The injections were performed at day 0 and day 7 , respecting a dose of $0.04 \mathrm{mmol} \mathrm{Gd} / \mathrm{kg}$ and $10 \mathrm{mg} \mathrm{PLP} / \mathrm{kg} /$ week. 


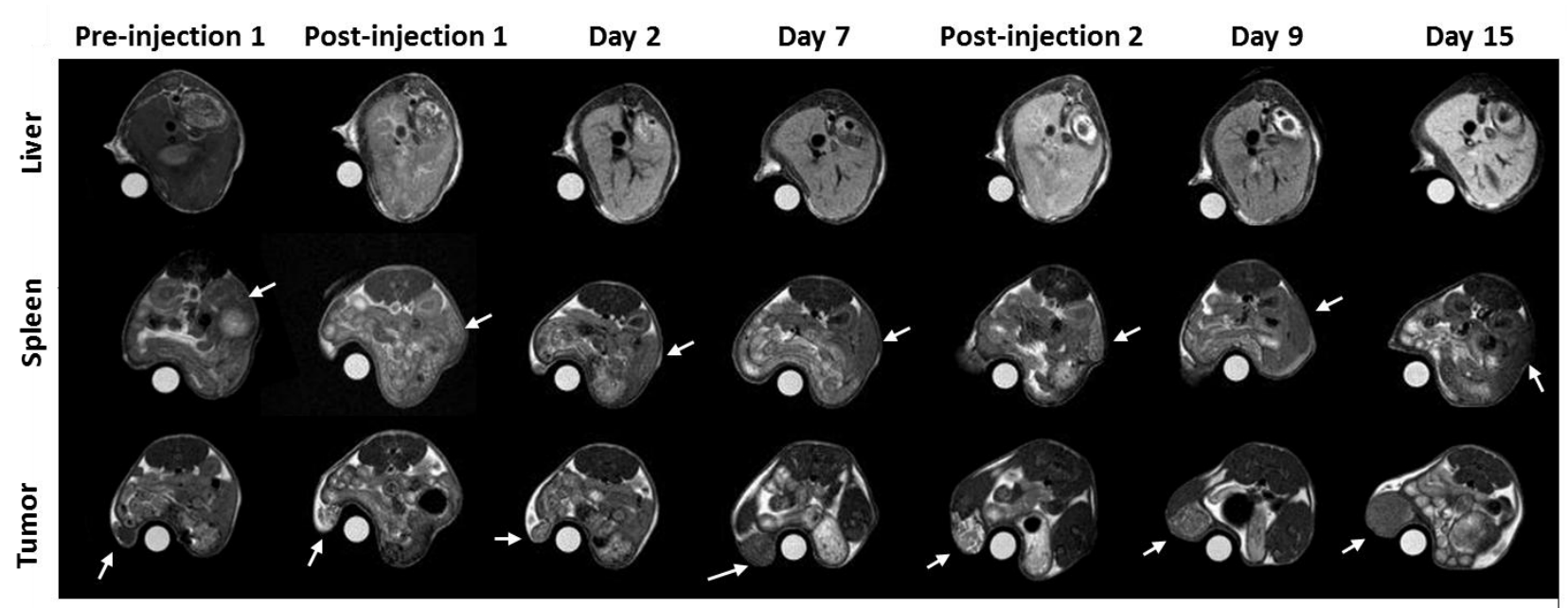

Figure 3. Representative MR images. Representative axial $T_{1 \mathrm{w}}$-images showing the evolution of $\mathrm{T}_{1}$ contrast over 15 days of MRI monitoring on liver (top), spleen (middle), and tumour (bottom) after the administration of Gd-PLP-DS.

As previously observed, ${ }^{10,14}$ the intratumour distribution of the contrast-enhanced areas appeared inhomogeneous, possibly reflecting local differences in the vascularization, which could affect the vesicular extravasation and the subsequent distribution of the contrast agents. After $24 \mathrm{~h}$ a similar $T_{1}^{E n h}$ value $(51.2 \%)$ was still calculated, whereas in the following days the contrast progressively decreased $\left(T_{1}^{E n h} \approx 30.7 \%\right.$ at day 7$)$. After the second administration, the $T_{1}{ }^{E N H}$ values followed again a similar trend, remaining stable for about $24 \mathrm{~h}$ and then declining progressively. Also the control PLP-Gd-LIPO displayed an equivalent ability to produce a positive signal in the tumour without any relevant difference in the time evolution of the contrast. Moreover, whereas in the muscular tissue no remarkable contrast enhancement was detected, the spleen and the liver showed substantial $T_{1}^{E n h}$ values that persisted for several days after the injection of both theranostic agents. This indicates the accumulation of the nanosystems in the macrophage-rich organs of the mononuclear phagocyte system (MPS). Furthermore, the imaging data obtained with PLP-Gd-DS show a good accordance with previous studies, where only a single administration of Gd-DS (0.05 mmol Gd/kg) was performed. ${ }^{10}$ Interestingly, after the second injection, the contrast in the liver 
increased over time, similarly to what has been recently reported in mice treated with liposomes loaded with Gadoteridol and Doxorubicin. ${ }^{21}$ Finally, the organs belonging to the excretory system (kidneys and bladder, Figure S2) were highlighted by a $\mathrm{T}_{1}$-contrast increase only immediately after the injection (bolus passage), thus excluding the renal filtration of the paramagnetic probe and confirming the good in vivo stability of the theranostic nanoparticles.

In vivo therapeutic effect

During the imaging sessions, anatomical $\mathrm{T}_{2 \mathrm{w}}$-images were also acquired to assess the tumour volumes and monitor the disease progression (Figure S3), thus enabling the evaluation of the effects of the drug transported by the nanocarriers and their overall therapeutic validity (Figure 4). Both PLP-loaded dendrimersomes and liposomes were capable of delaying the tumour progression with respect to the case of untreated animals. The two nanoformulations induced clear and statistically relevant therapeutic effects only by day 8 (Table S3), determining a final decrease in the tumour growth rate of $39.1 \%$ and $32.4 \%$ for PLP-Gd-DS and PLP-Gd-LIPO respectively, at day 15. At day 11 and 13, the increase in the tumour volume was slightly lower in mice receiving PLPGd-DS than in those receiving PLP-Gd-LIPO ( $p$-value $<0.05)$, whereas at the previous time points and at day 15 no substantial differences were detectable between the two groups. 


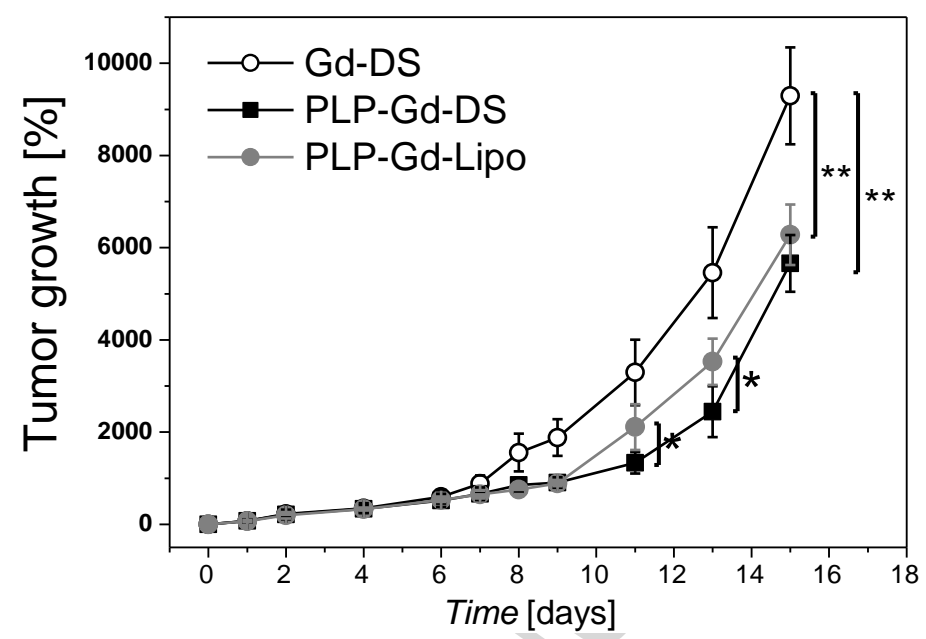

Figure 4. Tumour growth and in vivo therapeutic effect. Time course of the tumour growth expressed as the percentage volume increase with respect to the initial tumour volume calculated at time $0\left(20.4 \pm 1.5 \mathrm{~mm}^{3}\right)$, after the systemic administration of the control paramagnetic dendrimersomes (Gd-DS, white circles), the paramagnetic PLPloaded dendrimersomes (PLP-Gd-DS, black squares) or liposomes (PLP-Gd-LIPO, gray circles). Statistical significance addressed by ANOVA test (single and double asterisks indicating the $p$-value $<0.05$ and 0.01 , respectively).

\section{Dendrimersome biodistribution}

After the final MRI session (day 15), animals treated with PLP-Gd-DS were sacrificed to quantify the residual amount of Gadolinium in the different tissues (Figure 5). Organs and tumours were obtained also from a special additional cohort of diseased animals, which were sacrificed at day 7 without receiving the second dose of the theranostic dendrimersomes. As expected, a considerable metal content was detected in the sites displaying the highest MRI contrast (spleen, liver and tumour), whereas in the other investigated organs (kidney, muscle, heart, and lung) the amount of Gd was negligible both at day 7 and 15. On the one hand, the amount of Gd found in liver and tumour at day 15 was approximately three times greater than that measured at day 7; on the other hand, in the same time interval the metal content in the spleen increased to a much lesser extent. In the urine and blood collected immediately before the sacrifice, a sub-micromolar amount of metal was detected, which almost doubled from day 7 to day 15 . By considering that the concentration of the circulating $\mathrm{Gd}$ in a $19 \mathrm{~g}$ mouse at the moment of the first administration should approximately 
correspond to $506.7 \mu \mathrm{M}$ under our experimental conditions, one could estimate that after 7 days the metal concentration in blood is dramatically reduced by a factor of ca. 1700 .
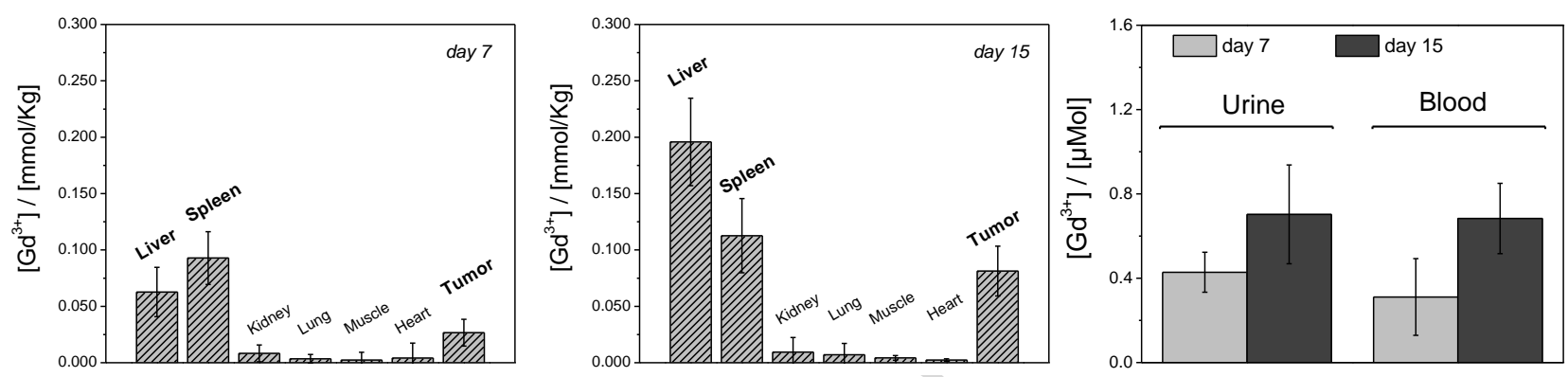

Figure 5. Residual gadolinium content in different tissues after the injection of PLP-Gd-DS. Amount of gadolinium normalized to the organ weight, measured by ICP-MS for different organs (liver, spleen, kidney, lung, muscle, and tumour) excised from mice at day 7 before the second administration of theranostic vesicles (left) or at day 15 after the last MRI acquisition (center). Gadolinium concentration in the blood and the urine immediately before the animals were sacrificed at day 7 and 15 (right).

\section{Discussion}

Stimulated by the fact that dendrimersomes could represent a relevant alternative to liposomes by virtue of the lower cost and the easy synthetic approach of the starting materials, ${ }^{10}$ the aim of the present study was to investigate the MRI theranostic potential of drug-loaded DSs to treat cancer. The choice of the tumour model and the therapeutic strategy relied on the previously reported ability of liposomal PLP to inhibit the B16.F10 melanoma growth, possibly through a series of distinct mechanisms of action. ${ }^{11-17}$ The experimental conditions selected for the in vivo theranostic scheme, including the time for initial tumor development (10 days) and that dedicated to the MRImonitoring (15 days), were defined on the basis of previous works ${ }^{11,22}$ and pilot experiments carried out on B16.F10-derived melanoma models. Although limited, a time window of around 2 weeks already proved to be sufficient to monitor and assess the therapeutic efficacy of PLP encapsulated in long-circulating liposomes, showing that the tumor growth inhibition could be observed after a single intravenous injection of $20 \mathrm{mg} / \mathrm{kg} /$ week in mice bearing melanoma B16 or colon carcinoma 
C26 xenografts. $^{23}$ In previous investigations, our group demonstrated the value of MRI in monitoring the activity of paramagnetic liposomes for this type of application, providing accuracy in the assessment of the therapeutic response to melanoma cancer. ${ }^{22}$ As a natural consequence, in this study the results obtained for the DSs were compared to a standard liposomal formulation endowed with similar physico-chemical characteristics. Based on in vitro tests, liposomes and DSs share a similar ability to load the PLP during preparation, and when used on cell lines they do not determine significantly different consequences on the viability, except for a slight increase of the cytotoxicity observed in case of the 6 h-long exposition to the liposome formulation. However, after one day of incubation this divergence vanished, so that the effect produced by the two nanoformulations became again equivalent and matched the cytotoxicity induced by the free PLP. Even the in vivo performance of the two systems either in terms of imaging contrast or therapeutic efficacy almost overlapped. Indeed, only at late stages of the protocol (day 11 and 13) the PLP-GdDS effectively provided some advantages in the anti-tumour efficacy as compared to the PLP-GdLIPO, but at the end of the experimental monitoring (day 15) such a difference turned out to be not statistical. Finally, the MRI contrast and the analytical quantification of gadolinium suggest a long lasting residence of the DSs into the tumour tissue and in the other accumulation sites commonly expected for nanoparticles, as spleen and liver. Particularly, an accumulation phenomenon was observed in the liver, possibly caused by the RES cell-mediated clearance of the nano-objects. This observation becomes more interesting when considering that, due to a reduced ability in producing pro-angiogenetic factors, the tumour-associated macrophages (TAMs) were already defined as crucial players in the biological response of B16.F10 melanoma to liposomal glucocorticoids. ${ }^{13}$ It has to be mentioned that the latest studies downgraded again the role of TAMs, by underlying a broader spectrum of PLP activities which includes also alterations of the tumour vasculature and dropdown of the circulating white blood cells, probably limiting the infiltration of angiogenesisstimulating monocytes. ${ }^{16,17}$ Nevertheless, based on our results, the marked cytotoxic activity induced by the PLP-Gd-DS and control liposomes on endothelial cells may support the previous 
data identifying the inhibition of the angiogenetic process as one of the leading therapeutic routes. Furthermore, the long-term retention of the nanoprobes in the RES-organs may raise reasonable safety concerns around the real biological applicability of this strategy, considering the potential PLP-induced toxicity towards hepatic and splenic tissue. In fact, even if rare, the occurrence of corticosteroid-induced acute liver injury has been already described in the literature. ${ }^{24}$ However, since the liposomal encapsulation makes more effective the drug delivery, the PLP carried by nanovectors is generally administrated at low dosages and well tolerated in vivo. ${ }^{12,25}$ In our experimental conditions, neither effects of acute toxicity were ever noticed on living animals during the monitoring time, nor gross alterations (such as infarct, necrosis, vacuolation) could be identified in the structure or morphology of the organs by visual inspection after extraction for the postmortem Gd quantification. Moreover, the intravital destiny of PLP after nanovectors-mediated uptake by the RES still has to be clarified in order to understand if the long-term MRI signal in liver and spleen could be ascribed to Gd-label deposition only, ${ }^{22}$ being thus not indicative of the drug presence. This issue and the potential side effects derived from non-specific PLP accumulation will be the objects for future research. Encouragingly in this perspective, a PLP liposomal formulation has been recently produced according to GMP qualitative standards, and may be readily used in humans subjects, as it turn out to be characterized by a very promising safety profile with no specific evidence of PLP systemic accumulation. ${ }^{26}$

\section{Conclusions}

In summary, the herein reported results indicate that 3,5-C12-EG-(OH)4 Janus dendrimers, loaded with a suitable paramagnetic Gd(III) complex to ensure MRI detection, are capable of encapsulating PLP with efficiency and retention ability similar to conventional liposomes. Importantly, also the anti-tumour activity and the MR imaging performance of the dendrimersomes are quite comparable to those observed for the liposomal carrier. However, it is important to highlight that DSs have some advantageous over liposomes mainly related to the low cost and the easy synthetic 
approach of the membrane constituents. In conclusion, the present report definitively highlights the great potential of dendrimersomes as valuable and cheaper alternative to liposomes for nanovesicles-based biomedical applications.

\section{Acknowledgements}

This work was supported by the European Union, project INMiND [grant number 278850, 2012], and by Compagnia di San Paolo (CSP-2014 THERASIL Project). 


\section{References}

[1]. V. Sanna, N. Pala, M. Sechi, Targeted therapy using nanotechnology: focus on cancer, Int. J. Nanomed. 9 (2014) 467-83.

[2]. M.F. Penet, B. Krishnamachary, Z. Chen, J. Jin, Z.M. Bhujwalla, Molecular imaging of the tumour microenvironment for precision medicine and theranostics, Adv. Cancer. Res. 124 (2014) 235-56.

[3]. S. Jeelani, R.C. Reddy, T. Maheswaran, G.S. Asokan, A. Dany, B. Anand, Theranostics: A treasured tailor for tomorrow, J. Pharm. Bioallied. Sci. 6 (2014) S6-8.

[4]. K.M. Bennett, J. Jo, H. Cabral, R. Bakalova, I. Aoki, MR imaging techniques for nanopathophysiology and theranostics, Adv. Drug Deliv. Rev. 74 (2014) 75-94.

[5]. M.S. Muthu, D.T. Leong, L. Mei, S.S. Feng, Nanotheranostics - application and further development of nanomedicine strategies for advanced theranostics, Theranostics 4 (2014) $660-77$.

[6]. M.E. Caldorera-Moore, W.B. Liechty, N.A. Peppas, Responsive theranostic systems: integration of diagnostic imaging agents and responsive controlled release drug delivery carriers, Acc. Chem. Res. 44 (2011) 1061-70.

[7]. B.T. Luk, R.H. Fang, L. Zhang, Lipid- and polymer-based nanostructures for cancer theranostics, Theranostics 2 (2012) 1117-26.

[8]. V. Percec, D.A. Wilson, P. Leowanawat, C.J. Wilson, A.D. Hughes, M.S. Kaucher, D.A. Hammer, D.H. Levine, A.J. Kim, F.S. Bates, K.P. Davis, T.P. Lodge, M.L. Klein, R.H. DeVane, E. Aqad, B.M. Rosen, A.O. Argintaru, M.J. Sienkowska, K. Rissanen, S. Nummelin, J. Ropponen, Self-assembly of Janus dendrimers into uniform dendrimersomes and other complex architectures, Science 328 (2010) 1009-14.

[9]. M. Filippi, J. Martinelli, G. Mulas, M. Ferraretto, E. Teirlinck, M. Botta, L. Tei, E. Terreno, Dendrimersomes: a new vesicular nano-platform for MR-molecular imaging applications, Chem. Commun. 50 (2014) 3453-3456. 
[10]. M. Filippi, D. Patrucco, J. Martinelli, M. Botta, P. Castro-Hartmann, L. Tei, E. Terreno, Novel stable dendrimersome formulation for safe bioimaging applications, Nanoscale 7 (2015) 12943-54.

[11]. M. Filippi, D. Remotti, M. Botta, E. Terreno, L. Tei, GdDOTAGA $\left(\mathrm{C}_{18}\right)_{2}$ : an efficient amphiphilic Gd(III) chelate for the preparation of self-assembled high relaxivity MRI nanoprobes, Chem. Commun. (Camb). 51 (2015) 17455-8.

[12]. R.M. Schiffelers, M. Banciu, J.M. Metselaar, G. Storm, Therapeutic application of longcirculating liposomal glucocorticoids in auto-immune diseases and cancer, J. Liposome Res. 16 (2006) 185-94.

[13]. M. Banciu, R.M. Schiffelers, M.H. Fens, J.M. Metselaar, G. Storm, Anti-angiogenic effects of liposomal prednisolone phosphate on B16 melanoma in mice, J. Control. Release 113 (2006) $1-8$.

[14]. M. Banciu, J.M. Metselaar, R.M. Schiffelers, G. Storm, Antitumour activity of liposomal prednisolone phosphate depends on the presence of functional tumour-associated macrophages in tumour tissue, Neoplasia 10 (2008) 108-17.

[15]. M. Banciu, M.H. Fens, G. Storm, R.M. Schiffelers, Antitumour activity and tumour localization of liposomal glucocorticoids in B16 melanoma-bearing mice, J. Control. Release 127 (2008) 131-6.

[16]. M. Banciu, J.M. Metselaar, R.M. Schiffelers, G. Storm, Liposomal glucocorticoids as tumourtargeted anti-angiogenic nanomedicine in B16 melanoma-bearing mice, J. Steroid. Biochem. Mol. Biol. 111 (2008) 101-10.

[17]. E. Kluza, S.Y. Yeo, S. Schmid, D.W. van der Schaft, R.W. Boekhoven, R.M. Schiffelers, G. Storm, G.J. Strijkers, K. Nicolay, Anti-tumour activity of liposomal glucocorticoids: The relevance of liposome-mediated drug delivery, intratumoural localization and systemic activity, J. Control. Release 151 (2011) 10-7. 
[18]. E. Kluza, M. Heisen, S. Schmid, D.W. van der Schaft, R.M. Schiffelers, G. Storm, B.M. ter Haar Romeny, G.J. Strijkers, K. Nicolay, Multi-parametric assessment of the anti-angiogenic effects of liposomal glucocorticoids, Angiogenesis 14 (2011) 143-53.

[19]. M.R. Mozafari, Liposomes: an overview of manufacturing techniques, Cell. Mol. Biol. Lett. (10) 2005, 711-9.

[20]. B.M. Mitruka, H.M. Rawnsley, Clinical Biochemical and Haematological Reference Values in Normal Experimental Animals and Normal Humans, Mosby, New York, 1981.

[21]. S. Rizzitelli, P. Giustetto, J.C. Cutrin, D. Delli Castelli, C. Boffa, M. Ruzza, V. Menchise, F. Molinari, S. Aime, E. Terreno, Sonosensitive theranostic liposomes for preclinical in vivo MRI-guided visualization of doxorubicin release stimulated by pulsed low intensity nonfocused ultrasound, J. Control. Release 202 (2015) 21-30.

[22]. E. Cittadino, M. Ferraretto, E. Torres, A. Maiocchi, B.J. Crielaard, T. Lammers, G. Storm, S. Aime, E. Terreno, MRI evaluation of the antitumour activity of paramagnetic liposomes loaded with prednisolone phosphate, Eur. J. Pharm. Sci. 45 (2012) 436-41.

[23]. R.M. Schiffelers, J.M. Metselaar, M.H.A.M. Fens, A.P.C.A. Janssen, G. Meloma, G. Storm, Liposome-encapsulated prednisolone phosphate inhibits growth of established tumors in mice, Neoplasia. 7 (2005) 118-127.

[24]. P. Carrier, B. Godet, S. Crepin, L. Magy, M. Debette-Gratien, B. Pillegand, J. Jacques, D. Sautereau, E. Vidal, F. Labrousse, G. Gondran, V. Loustaud-Ratti, Acute liver toxicity due to methylprednisolone: consider this diagnosis in the context of autoimmunity, Clin. Res. Hepatol. Gastroenterol. 37 (2013) 100-4.

[25]. J.M. Metselaar, M.H. Wauben, J.P. Wagenaar-Hilbers, O.C. Boerman, G. Storm, Complete remission of experimental arthritis by joint targeting of glucocorticoids with long-circulating liposomes, Arthritis Rheum. 48 (2003) 2059-66.

[26]. M.E. Lobatto, C. Calcagno, M.J. Otten, A. Millon, S. Ramachandran, M.P. Paridaans, F.M. van der Valk, G. Storm, E.S. Stroes, Z.A. Fayad, W.J. Mulder, J.M. Metselaar, 
Pharmaceutical development and preclinical evaluation of a GMP-grade anti-inflammatory nanotherapy, Nanomedicine. 11 (2015) 1133-40. 


\section{Graphical abstract}

Dendrimersomes loaded with both the MRI reporter GdDOTAGA $\left(\mathrm{C}_{18}\right)_{2}$ and the glucocorticoid drug Prednisolone Phosphate (PLP) displayed in vitro and in vivo efficacy in the treatment of murine melanoma.

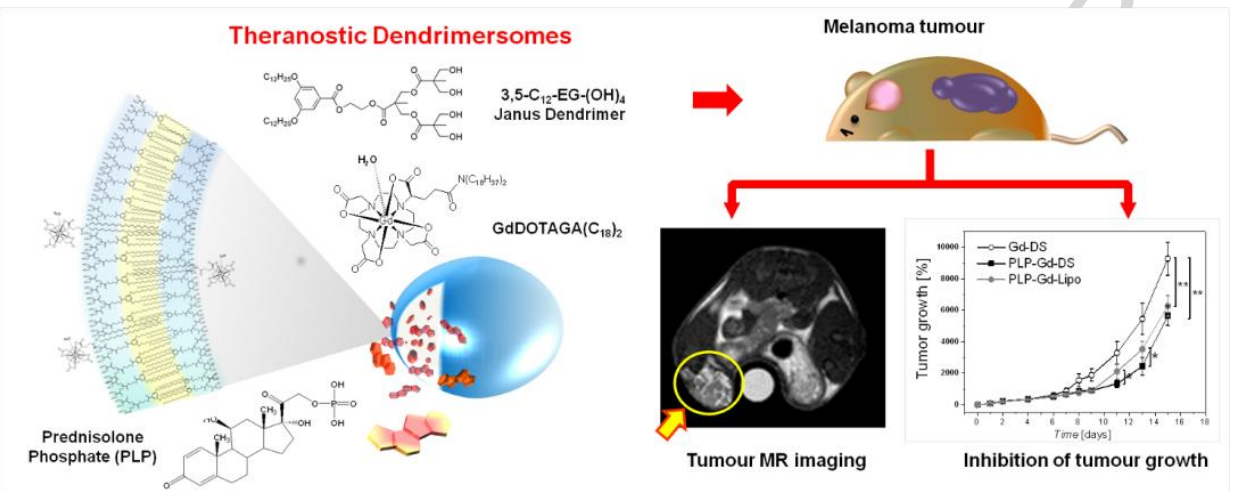

